\title{
Crestal Bone Changes in Different Implants Designs: A Prospective Clinical Trial
}

\author{
${ }^{1}$ Department of Dentistry, Lutheran University of Brazil, Canoas-RS, \\ Brazil \\ 2Department of Dentistry, Federal University of Santa Catarina, \\ Florianópolis-SC, Brazil
}

\begin{abstract}
Address for correspondence Luis André Mezzomo, DDS, PhD, Department of Dentistry, Federal University of Santa Catarina, Delfino Conti, S/N, Health Sciences Center, Trindade, Florianópolis, Brazil (e-mail: Imezzomo@hotmail.com).
\end{abstract}

Eur J Dent 2019;13:497-502

\begin{abstract}
Keywords

- bone level

- dental implant

- marginal bone

- tissue level

Objective Several modifications, such as changes in the implant-abutment connection, have been suggested in studies on dental implants to better preserve the peri-implant bone level. The aim of this study was to prospectively compare crestal bone level changes between two different implant designs-tissue level ( $\mathrm{TL}$ ) and bone level (BL).

Materials and Methods The sample comprised 18 patients, on whom a total of 30 hydrophilic tissue- and bone level implants were placed (SLActive, Straumann Institut AG, Basel, Switzerland), in the posterior region of the maxilla or the mandible. Impressions were taken after 45 days of healing. Then, cemented-retained metalloceramic crowns were fabricated and installed. Marginal bone level changes were assessed by the paralleling technique of periapical radiographies, on both mesial and distal aspects of each implant, at the moment of the implant placement and after 1 year of loading. Photoshop software was used to perform linear measurements by a single and calibrated examiner.

Statistical Analysis The Mann-Whitney test at a $5 \%$ significance level was used to compare the bone changes among the implants assessed.

Results A significantly lower $(p=0.048)$ bone remodeling was observed on bone level implants $(0.05 \mathrm{~mm})$, when compared to tissue level implants $(0.47 \mathrm{~mm} ; p=0.048)$. The average marginal bone level changes at the distal aspect did not show any statistically significant difference $(p=0.325)$.

Conclusions Tissue level implants presented greater bone loss in the mesial surface than bone level implants. Both designs presented stable and clinically acceptable bone crests.
\end{abstract}

\section{Introduction}

The maintenance of stable marginal and peri-implant bone tissues plays a decisive role on the long-term success of dental implants. ${ }^{1-5}$ For hexagonal platform implants, it has been stated that a bone remodeling of about 1 to $1.5 \mathrm{~mm}$ can be expected in the first year of loading, whereas in the subsequent years, the annual bone loss rate might be about $0.2 \mathrm{~mm}$. ${ }^{6}$ Bone remodeling might be associated to factors such as surgical trauma, the formation of the peri-implant biological width, the position of the rough surface of an implant on a smooth machined collar in relation to the bone, or bacterial contamination due to the presence of a microgap between the prosthetic abutment and the implant platform. ${ }^{7.8}$

Several modifications, such as changes in the implantabutment connection, have been suggested in studies on dental implants in order to better preserve the peri-implant bone level. ${ }^{9}$ The Straumann Dental Implant System offers two different implant designs: tissue level (TL) implants have an internal conical connection, and their prosthetic platform is intended to vertically put away the microgap between the implant platform and the prosthetic abutment, as well as 
possible inflammatory cells. Bone level (BL) implants have an internal Morses taper implant-abutment connection, especially designed to evenly distribute the stress patterns to the surrounding bone. In BL implants, the nonmatched (platform switching) connection shows a better capacity to produce biological formation, as the microgap is horizontally distanced from the bone (differently from flat-to-flat connections [i.e., external hexagon] or matched diameters connections). This type of connection enables an adequate biological sealing, while limiting bacterial infiltration. ${ }^{10}$ In spite of the growing evidences of the efficacy of both implant design concepts, there are still few direct comparisons of their capacities to clinically preserve the marginal bone levels. Therefore, the aim of this study is to assess crestal bone change in two different implant designs-tissue level and bone level-through radiography and prospective comparison.

\section{Materials and Methods}

\section{Null Hypothesis}

Different implant designs from the same manufacturer and with equal surface characteristics, implant length, and diameter present similar crestal bone levels after 1 -year of loading.

\section{Study Design and Ethical Considerations}

This was designed as a prospective observational study. The study was approved by the Local Ethics Research Committee of the Lutheran University of Brazil (CEP-ULBRA 2010-526H), and was conducted in accordance with the Declaration of Helsinki. Patients were asked to sign a written consent form in order to participate in the study.

\section{Study Population}

Patients were selected through anamnesis, according to the eligibility criteria set by the classification of the American Society of Anesthesiologists (ASA Classification), followed by intraoral clinical examination, and radiographic examination (CBCT scans). One or more implants were applied to each patient, according to the individual necessity and considering the bone availability.

\section{Eligibility Criteria \\ Inclusion Criteria}

The study included male and female patients aged from 18 to 75 years old; in good general health (classified as ASA 1 or ASA 2); partially edentulous and in need of dental implants in single sites adjacent to natural teeth, in the posterior region of the maxilla or the mandible, with one or more implants per arch, depending on the case.

\section{Exclusion Criteria}

The study did not include pregnant or lactating patients; patients under anticoagulants therapy; patients under bisphosphonates therapy; patients with metabolic diseases (except controlled diabetes mellitus), hepatitis, or psychological disorders; smokers; nontreated periodontal disease; patients with severe bruxism (clear clinical signs and symptoms); and patients with acute endodontic lesions in adjacent areas to the implant site or with areas of previous bone augmentation.

\section{Surgical Procedure}

Implant sites were chosen as to not allow the placement of two adjacent implants. The implants were placed either between neighboring teeth or as a single implant distal to the last tooth in the arch. The surgeries were performed by a single and calibrated operator. The surgical procedure was performed after asepsis of the patient's mouth and local anesthesia, with a linear mid-crestal incision and the elevation of a full-thickness flap. Then, the bone site was drilled under constant irrigation with saline, in low speed and with the sequence of burs, as recommended by the manufacturer. Lastly, the implant was placed in accordance with the treatment plan.

No bone augmentation was performed in any of the study implants. The implants used were $8 \mathrm{~mm}$-long hydrophilic (SLActive) tissue level standard plus (RN; ø4.1 mm/ø4.8 mm, matching platforms), and bone level (RC; ø4.1 mm, platform switching; Straumann Institut AG, Basel, Switzerland;

\section{-Fig. 1).}

Tissue level implants had their junction between the smooth collar and the rough surface of the body placed at the level of the bone crest. The $1.8 \mathrm{~mm}$-smooth collar was positioned entirely above the bone crest. Due to differences in the heights of bone walls, because of adjacent dental absence, some cases demanded the positioning of a machined intraosseous collar in the proximal aspects. Bone level implants were all placed at the bone crest level.

After the implant placement, $2 \mathrm{~mm}$-high healing abutments were placed on top of it, and the full-thickness flap was repositioned and stabilized with single interrupted sutures (one-stage approach). All patients were prescribed
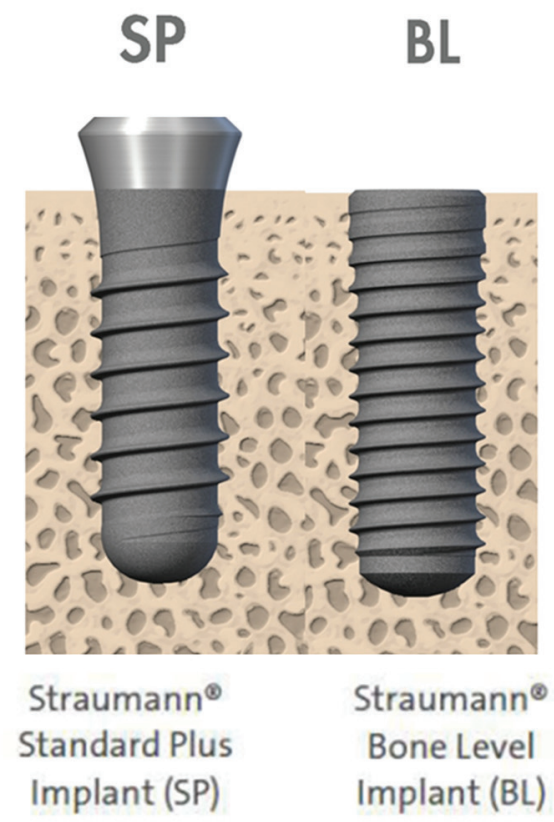

Fig. 1 Image of Straumann tissue and bone level implants, respectively. 
$500 \mathrm{mg}$ amoxicillin every 8 hours for 7 days, $750 \mathrm{mg}$ paracetamol every 6 hours for 2 days, and $0.12 \%$ chlorhexidine digluconate rinses twice a day for 7 days, as a postoperative care protocol. Patients allergic to amoxicillin were prescribed clindamycin 500 mg every 8 hours for 7 days. Oral hygiene instructions and mastication precautions were given to all patients.

\section{Radiographic Assessment}

The equipment used was composed of a long-cone paralleling technique for digital periapical radiography, with the aid of the Rinn XCP x-rays holder (Dentsply, United States), and a Timex 70E x-ray machine (Gnatus Medical Dental Equipment Ltda, Ribeirão Preto, Brazil), with an exposure time of 0.4 seconds. Bite blocks were customized for each patient with the aid of a low contraction red acrylic resin (Duralay, Reliance Dental MFG. Co, United States). The intraoral digital x-rays system Gnatus DRS and the Cygnus Imaging software (Gnatus Medical Dental Equipment Ltda, Ribeirão Preto, Brazil) for Windows were used in order to obtain and to visualize images. Baseline radiography was taken shortly after the implant placement.

\section{Prosthetic Procedures}

After 45 days of healing (early loading), impressions were taken and metalloceramic crowns were fabricated. The Synocta abutment and the anatomical abutment (Straumann Institut AG, Basel, Switzerland), both for cemented-retained restorations, were used for tissue-level and for bone level implants, respectively. The crowns were cemented with provisional cement Rely-X Temp, 3M (Deutschland; Seefeld, Germany).

For the bone level implants, the anatomic abutments were prepared in order to place the cervical margin of the metalloceramic crowns at a $1.8 \mathrm{~mm}$ distance from the bone crest. This allowed the standardization of the vertical distance between the crown and the abutment, in both implant designs (tissue level and bone level), excluding thus any bias.

After 1 year of loading, new radiographies were taken using the same technique and the customized bite blocks that were previously described. The prosthetic crowns were removed for the examination and recemented afterwards.

Baseline and 1-year post-loading radiographic examinations ( - Fig. 2) allowed linear measurements by means of a Photoshop software (San Jose; California, United States), as well as a comparison between the two implant designs. The procedure of the measurement was based in a preview study. ${ }^{11}$

The distance measured was from the implant platform to the most coronal point of the bone crest, at mesial and distal aspects. This measurement was performed twice on each image, by the same calibrated examiner, in order to assure their precision.

\section{Statistical Analysis}

Upon checking the normality of the data, the Mann-Whitney nonparametric test was applied in order to verify whether there were differences between the groups, at a $5 \%$ significance level. The implant was considered as the unit of analysis. The percentile was used as a descriptive statistical
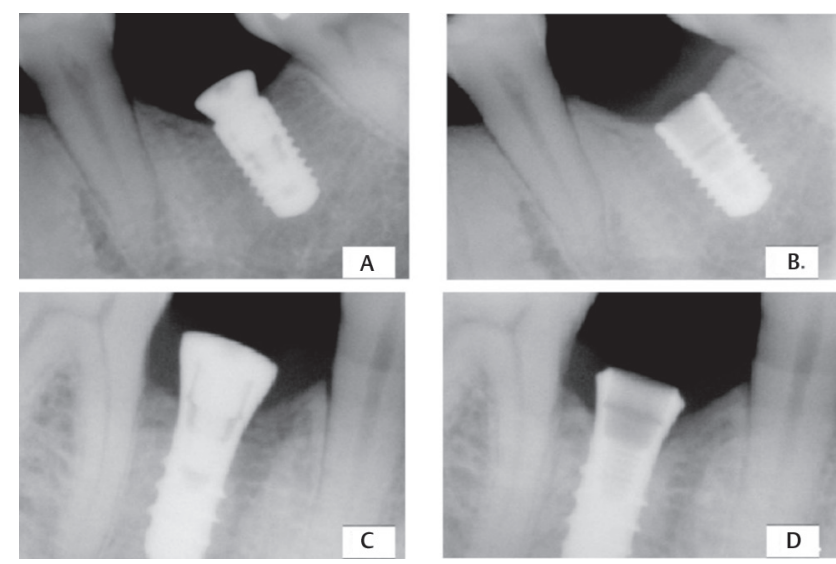

Fig. 2 (A) Time of installation (BL), (B) 1-year of implant in function (BL), (C) installation time (TL), and (D) 1-year of implant in function (TL). BL, bone level; TL, tissue level.

measure. To permit that, the data were grouped in an increasing way. Both types of implants were analyzed regarding the average bone remodeling, the standard deviation, minimum and maximum values, and the percentile. Due to asymmetries, data variability and sample sizes, the median and interquartile range (P25; P75) will be presented as descriptive data.

\section{Results}

Eighteen patients (12 women and 6 men, aged between 25 and 68 years old [average age: 53.17 years]) met the eligibility criteria and were selected for implant placements. Two patients were not followed up in the study due to external reasons. The study followed up 27 out of the 30 implants placed-13 bone level and 14 tissue level implants. Neither postoperative biological nor prosthetic complications were recorded after implant placement and prosthetic reconstruction. The sites of the implants were: $10 \mathrm{BL}$ implants in posterior mandible, three BL implants in the posterior maxilla, nine TL implants in posterior mandible, and five TL implants in the posterior maxilla.

When comparing the mesial aspects, it was possible to observe a significant difference in the marginal bone levels between the two types of prosthetic platforms. A significantly lower crestal bone change ( $p=0.048$ ) was observed on bone level implants $(0.05 \mathrm{~mm})$ in comparison to those occurred in tissue level implants $(0.47 \mathrm{~mm})$. When the comparison was performed in the distal aspects of these implants, no statistically significant difference $(p=0.325)$ was observed between the two types of implant ( - Fig. 3 ).

The greatest crestal bone change in the mesial aspect in bone level implants was $0.83 \mathrm{~mm}$, and the maximum bone gain was $+1.01 \mathrm{~mm}$. In the distal aspect, the maximum bone loss on bone level implants was $1.53 \mathrm{~mm}$, and the maximum bone gain was $+0.43 \mathrm{~mm}$.

In tissue level implants, the greatest crestal bone in the mesial aspect level change was $1.78 \mathrm{~mm}$, and the maximum was $+0.64 \mathrm{~mm}$. In the distal aspect, the maximum bone loss was $2.09 \mathrm{~mm}$, and the maximum bone gain was $+0.6 \mathrm{~mm}$. 

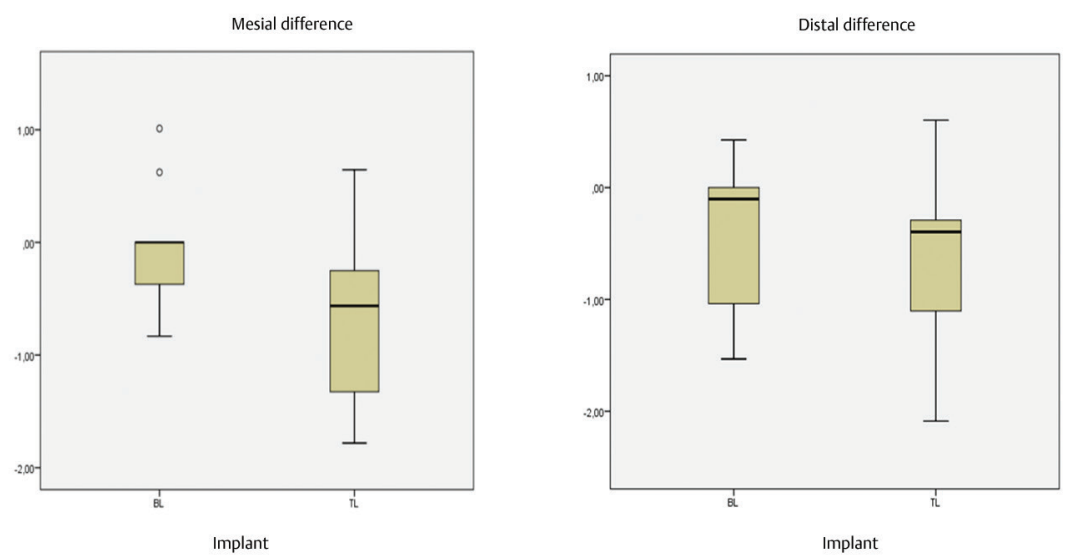

Fig. 3 Comparison between the mesial and distal bone behaviors of the BL implant with the TL implant through the median and the interquartile range (P25; P75). BL, bone level; TL, tissue level.

\section{Discussion}

Physiological remodeling around dental implants has been widely reported in the literature. A large part of the studies is focused on the types of implant (single-piece vs. two-pieces) or on the type of abutment, (especially in two-piece implants cases). Our study included two types of two-pieces implants: the bone level implant, installed at the level of the marginal bone, and the tissue level implant, installed at the level of the soft tissue. Both implant systems have a similar external intraosseous surface. The remodeling values found in the 1-year follow-up were comparable to other studies ${ }^{12,13}$ and were considered as acceptable levels, according to the criterion of a crestal bone change being lower than $0.5 \mathrm{~mm} .{ }^{1}$

A retrospective study ${ }^{13}$ assessed 337 implants and found higher average crestal level bone change for tissue level implants. The average marginal bone losses found for bone level implants were $0.3,0.38$, and $0.48 \mathrm{~mm}$, whereas for tissue level implants, the average bone losses were of $0.6,0.54$, and 0.93 , in follow-up periods of 12,24 , and 36 months, respectively. Although there was no statistically significant difference between the two groups in the periods of 6 to 12 months after the placement of implants, there was a slightly higher amount of changes in the level of bone in tissue level implants than in bone level implants.

The implants assessed exposed differences between the mesial aspects in the bones behavior. Bone level implants showed better cervical preservation than tissue level implants in the mesial region, as shown in radiographic images. When the comparison was made in the distal aspect of these implants, the crestal bone behavior was similar.

The lower crestal remodeling in the mesial region presented in the bone level implants can be explained by the greater precision that this method allows in limiting the margin of the treated surface of the implant. The tissue level implant, on the other hand, involves a machined collar, and the intraosseous placement of the smooth-rough interface causes a greater loss in the region. ${ }^{12,14}$
Due to the sites chosen for implant placement in our study, the distal regions could remain untouched, but not the mesial, since there was sometimes an intrabony positioning of the machined collar in the mesial regions in some tissue level implants. Larger bone remodeling may have occurred due to the establishment of the peri-implant biological space, once this causes a lack of interaction between the smooth surface and the bone tissue. ${ }^{15}$ In bone level implants, there is no machined collar, which facilitates the visualization of the implant platform to accomplish the placement in the bone level.

A preestablished distance of $1.8 \mathrm{~mm}$ from the interface crown/prosthetic abutment to the bone in bone level implants standardized the distance between the metal-ceramic crowns and the bone margins, for both kinds of implant. This allowed to comparing the presence of the peri-implant space and the infiltration of inflammatory cells between the two types of implant, as the distances were equal. Therefore, it was possible to evaluate the relation between the abutment connection and the bone level implant per se in relation to marginal bone behavior. This type of platform, where the microgap is horizontally distanced from the bone crest, did not cause greater bone loss ${ }^{16}$ than the ones caused by tissue level implants, which is the type used as a control in many animal studies for bone loss evaluation (through radiographic and histological control). ${ }^{1718}$ The present results confirm the crestal bone maintenance capability of bone level implants. ${ }^{18}$ The positioning of the prosthetic platform at the bone level is important for the crestal bone maintenance. When it is intraosseously positioned, a greater bone resorption can be expected. ${ }^{19-22}$

A systematic review ${ }^{23}$ compared marginal changes of bone level in crestal bones in platform switching (PS) and in platform matching (PM) methods for dental implant. In that study, controlled clinical trials were randomly included in order to compare one or more PS groups with one or more PM groups, with at least 12 months of follow-up after loading. Fifteen publications, involving a total of 1,439 implants and 642 patients, were eligible. Most studies with PS implants 
showed a bone remodeling, while none of them showed differences in terms of implant failure rates. In another systematic review comparing implants with and without the switching platform for tissue level implants, similar marginal bones behavior was observed among the control (tissue level) and the platform switching groups, and a greater cervical bone loss was observed in them when compared to implants without platforms. ${ }^{24,25}$

Although bone remodeling occurred when the machined collar of the tissue level implants was placed intraosseously, it did not cause problems, since the resorption occurred basically above the treated surface region, maintaining $8 \mathrm{~mm}$ length for the implant with bone support. As previously reported by other studies, crestal bone maintenance in these two types of implants is very successful, and widely accepted as a reference for this aspect. ${ }^{18,26}$

The production of radiographic evaluations from the moment of placing the implant was important in order to evaluate the initial bone remodeling, which typically occurs due to aspects such as surgical trauma or breaking of the homeostasis of soft and hard tissues. ${ }^{7}$ The 1-year follow-up time for this study was set since this is the interval when the greatest bone resorption is expected to occur.

\section{Conclusion}

The tissue level implant presented greater bone loss on the mesial surface than the bone level; however, both designs presented stable and clinically acceptable bone crests.

\section{Conflict of Interest}

None declared.

\section{References}

1 Albrektsson T, Zarb G, Worthington P, Eriksson AR. The longterm efficacy of currently used dental implants: a review and proposed criteria of success. Int J Oral Maxillofac Implants 1986;1(1):11-25

2 Lekholm U, Gunne J, Henry P, et al. Survival of the Brånemark implant in partially edentulous jaws: a 10 -year prospective multicenter study. Int J Oral Maxillofac Implants 1999;14(5):639-645

3 Brocard D, Barthet P, Baysse E, et al. A multicenter report on 1,022 consecutively placed ITI implants: a 7-year longitudinal study. Int J Oral Maxillofac Implants 2000;15(5):691-700

4 Östman P-O, Hellman M, Sennerby L. Ten years later. Results from a prospective single-centre clinical study on 121 oxidized (TiUnite ${ }^{\mathrm{TM}}$ ) Brånemark implants in 46 patients. Clin Implant Dent Relat Res 2012;14(6):852-860

5 Rabel A, Köhler SG, Schmidt-Westhausen AM. Clinical study on the primary stability of two dental implant systems with resonance frequency analysis. Clin Oral Investig 2007;11(3):257-265

6 Albrektsson T, Zarb GA. Current interpretations of the osseointegrated response: clinical significance. Int J Prosthodont 1993;6(2):95-105

7 Cochran DL, Nummikoski PV, Higginbottom FL, Hermann JS, Makins SR, Buser D. Evaluation of an endosseous titanium implant with a sandblasted and acid-etched surface in the canine mandible: radiographic results. Clin Oral Implants Res 1996;7(3):240-252

8 Buser D, Nydegger T, Oxland T, et al. Interface shear strength of titanium implants with a sandblasted and acid-etched surface: a biomechanical study in the maxilla of miniature pigs. J Biomed Mater Res 1999;45(2):75-83

9 Lin M-I, Shen Y-W, Huang H-L, Hsu J-T, Fuh L-J. A retrospective study of implant-abutment connections on crestal bone level. J Dent Res 2013;92(12Suppl :202S-207S

10 Mangano C, Mangano F, Piattelli A, Iezzi G, Mangano A, La Colla L. Prospective clinical evaluation of 1920 Morse taper connection implants: results after 4 years of functional loading. Clin Oral Implants Res 2009;20(3):254-261

11 Ganeles J, Zöllner A, Jackowski J, ten Bruggenkate C, Beagle J, Guerra F. Immediate and early loading of Straumann implants with a chemically modified surface (SLActive) in the posterior mandible and maxilla: 1-year results from a prospective multicenter study. Clin Oral Implants Res 2008;19(11):1119-1128

12 Valderrama P, Bornstein MM, Jones AA, Wilson TG, Jr. Higginbottom FL, Cochran DL. Effects of implant design on marginal bone changes around early loaded, chemically modified, sandblasted Acid-etched-surfaced implants: a histologic analysis in dogs. J Periodontol 2011;82(7):1025-1034

13 Kumar VV, Sagheb K, Kämmerer PW, Al-Nawas B, Wagner W. Retrospective clinical study of marginal bone level changes with two different screw-implant types: comparison between tissue level (TE) and bone level (BL) implant. J Maxillofac Oral Surg 2014;13(3):259-266

14 Hartman GA, Cochran DL. Initial implant position determines the magnitude of crestal bone remodeling. J Periodontol 2004;75(4):572-577

15 Broggini N, McManus LM, Hermann JS, et al. Persistent acute inflammation at the implant-abutment interface. J Dent Res 2003;82(3):232-237

16 Lazzara RJ, Porter SS. Platform switching: a new concept in implant dentistry for controlling postrestorative crestal bone levels. Int J Periodontics Restorative Dent 2006;26(1):9-17

17 Weber HP, Crohin CC, Fiorellini JP. A 5-year prospective clinical and radiographic study of non-submerged dental implants. Clin Oral Implants Res 2000;11(2):144-153

18 Heitz-Mayfield LJ, Darby I, Heitz F, Chen S. Preservation of crestal bone by implant design. A comparative study in minipigs. Clin Oral Implants Res 2013;24(3):243-249

19 Alomrani AN, Hermann JS, Jones AA, Buser D, Schoolfield J, Cochran DL. The effect of a machined collar on coronal hard tissue around titanium implants: a radiographic study in the canine mandible. Int J Oral Maxillofac Implants 2005; 20(5):677-686

20 Jung RE, Jones AA, Higginbottom FL, et al. The influence of non-matching implant and abutment diameters on radiographic crestal bone levels in dogs. J Periodontol 2008;79(2):260-270

21 Hansson S, Norton M. The relation between surface roughness and interfacial shear strength for bone-anchored implants. A mathematical model. J Biomech 1999;32(8):829-836

22 Hänggi MP, Hänggi DC, Schoolfield JD, Meyer J, Cochran DL, Hermann JS. Crestal bone changes around titanium implants. Part I: a retrospective radiographic evaluation in humans comparing two non-submerged implant designs with different machined collar lengths. J Periodontol 2005;76(5):791-802

23 DI Girolamo M, Calcaterra R, DI Gianfilippo R, Arcuri C, Baggi L. Bone level changes around platform switching and platform matching implants: a systematic review with meta-analysis. Oral Implantol (Rome) 2016;9(1):1-10

24 Atieh MA, Ibrahim HM, Atieh AH. Platform switching for marginal bone preservation around dental implants: 
a systematic review and meta-analysis. J Periodontol 2010;81(10):1350-1366

25 King GN, Hermann JS, Schoolfield JD, Buser D, Cochran DL. Influence of the size of the microgap on crestal bone levels in non-submerged dental implants: a radiographic study in the canine mandible. J Periodontol 2002;73(10):1111-1117
26 Astrand P, Engquist B, Dahlgren S, Gröndahl K, Engquist E, Feldmann $\mathrm{H}$. Astra Tech and Brånemark system implants: a 5 -year prospective study of marginal bone reactions. Clin Oral Implants Res 2004;15(4):413-420 